\title{
Optimization of Hierarchical Modulation for Use of Scalable Media
}

\author{
Yongheng Liu ${ }^{1}$ and Conor Heneghan ${ }^{2}$ \\ ${ }^{1}$ Department of Electronic and Electrical Engineering, University College Dublin, Dublin, Ireland \\ ${ }^{2}$ Communication Digital Signal Processing Group, National University of Ireland, Dublin, Ireland
}

Correspondence should be addressed to Yongheng Liu, yongheng.liu@gmail.com

Received 2 August 2009; Revised 3 January 2010; Accepted 13 January 2010

Academic Editor: Ling Shao

Copyright ( $) 2010$ Y. Liu and C. Heneghan. This is an open access article distributed under the Creative Commons Attribution License, which permits unrestricted use, distribution, and reproduction in any medium, provided the original work is properly cited.

This paper studies the Hierarchical Modulation, a transmission strategy of the approaching scalable multimedia over frequencyselective fading channel for improving the perceptible quality. An optimization strategy for Hierarchical Modulation and convolutional encoding, which can achieve the target bit error rates with minimum global signal-to-noise ratio in a single-user scenario, is suggested. This strategy allows applications to make a free choice of relationship between Higher Priority (HP) and Lower Priority (LP) stream delivery. The similar optimization can be used in multiuser scenario. An image transport task and a transport task of an H.264/MPEG4 AVC video embedding both QVGA and VGA resolutions are simulated as the implementation example of this optimization strategy, and demonstrate savings in SNR and improvement in Peak Signal-to-Noise Ratio (PSNR) for the particular examples shown.

\section{Introduction}

Recent developments in media source coding have evolved from consideration not only of compression efficiency in terms of rate-distortion curves, but also on methods for providing easy-to-use scalability features. Scalability refers to the ability of the media delivery system to easily provide a range of spatial, temporal, and quality profiles in response to changing system conditions or user demands. For example, a person viewing a sports event on a mobile phone may be content to view a QCIF $(176 \times 144$ pixels $)$ resolution level at $25 \mathrm{fps}$, whereas a person with access to an HDTV may wish for a $50 \mathrm{fps}, 720 \mathrm{p}(1280 \times 720$ pixels $)$ version of the same media. Such demands can be met using scalable video and audio coding, where lower resolution or lower quality signals can be reconstructed from partial bit streams. This allows simpler delivery of digital media, as networks and terminals can autonomously adapt to issues such as network heterogeneity and error-prone environments (e.g., wireless fading channels) [1]. Scalability allows the removal of parts of the bitstream, while achieving a rate-distortion
(R-D) performance with the remaining partial bitstream (at any supported spatial, temporal, or SNR resolution), that is, comparable to a "single-layer" approach [2], that is, nonscalable H.264/MPEG-4 AVC coding (at that particular resolution) [3].

However, in order to take maximum advantage of scalable coding, we need to ensure that scalability is treated at a system level, so that all layers of the communication stack can make intelligent decisions about how to use scalability. For example, in real-time audio-visual traffic, consecutive packets carry data of different importance for the user perceived quality. Header information is of vital importance, whereas texture information (in video coding) can tolerate some errors. So, although data may be lost due to congestion or poor wireless channel conditions, the class of data lost will have the largest impact on user experience [4]. Nevertheless, many current media transmission systems assume all data from higher layers is equal in importance, and rely upon the higher layers to provide the additional redundancy which can help protect more important information. However, it can be agreed that scalable media codecs often have the inherent 
property that some data is more important than others, and exploiting that knowledge may enhance overall system performance.

One strategy that could be employed is to use timeslicing of data with different priorities; however in [5], Cover proved that if a sender wants to send information simultaneously to several receivers, given specific channel conditions, superimposing high-rate information on lowrate information may achieve higher bandwidth efficiency than the time-sharing strategy.

This has led to the concept of an alternative approach for dealing with different streams of information at the physical layer of a system, namely, hierarchical modulation. Hierarchical Modulation (known also as embedded or multiresolution modulations) is one of the ways to implement the superimposition of multichannel signals, which uses constellations with nonuniformly spaced signal points. Many researchers have shown interest in this strategy [6-8]. Normally, two or more separate data streams are modulated onto one single constellation symbol stream, as shown in Figure 1. The two classes of data can also be treated using channel coding with different code rates in order to cope with channel noise and fading. By tuning the code rate, a tradeoff of bit rate and bit error probability can be achieved. This concept was studied further in the early nineties for digital video broadcasting systems $[8,9]$, and has gained more interest recently with the demand to support multimedia services by simultaneous transmission of different types of traffic, each with its own quality requirement [10-12]; and a possible application in the DVB-T standard [13] in which hierarchical modulations can be used on OFDM sub-carriers. A two-level hierarchical modulation scheme is an optional transmission feature of the DVB-T system, in which the data to broadcast is split into two parts: a high priority (HP) stream with a strong protection against errors, and a low priority (LP) one with less protection. Receivers with "good" reception conditions (e.g., closer to the transmitter and/or with higher antenna gains) can receive both streams, while those with poorer reception conditions may only receive the "High Priority" stream. Broadcasters can target two different types of DVB-T receiver with two completely different services. Typically, the LP stream is of a higher bit rate, but lower robustness than the HP one. For example, a broadcast could choose to deliver HDTV in the LP stream. The implementation of hierarchical modulation in the Digital Video Broadcast standard for terrestrial broadcast (DVB-T) in Europe [13] is a typical two services for two users scenario. Its main purpose is to provide two types of service (HDTV and SDTV), to carry multiple programs, and to increase capacity $[14,15]$.

Scalable coding interacts naturally with hierarchical modulation. Since the packets encoded by scalable codecs can be divided into different classes of priority, a simple scheme would create two classes such as "base information" and "refinement information" according to their contribution to the quality/temporal/spatial resolution of the media. The packets belonging to the base level can be allocated to the base bits of the hierarchial constellation, meanwhile the refinement packets can be assigned to the refinement bits of

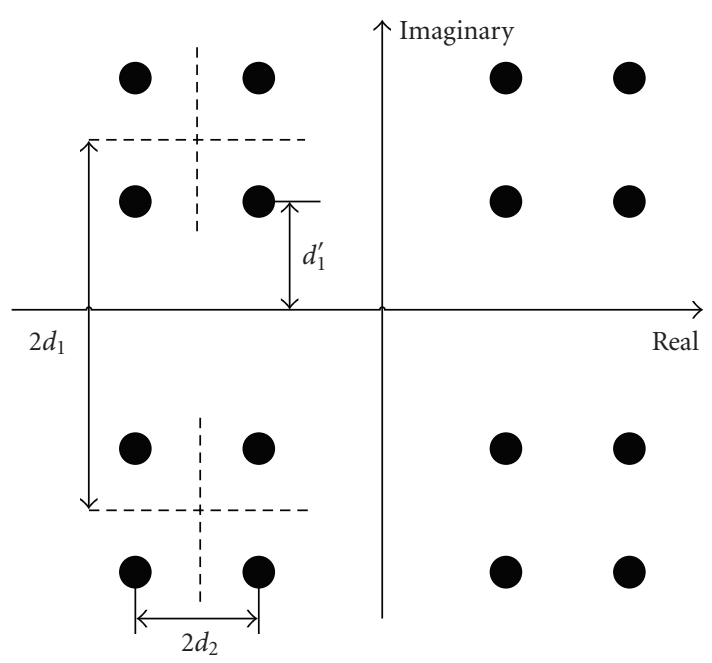

FIgure 1: A 16-QAM constellation used in Hierarchical Modulation.

the constellation. The user who is able to decode the base bits of the hierarchical constellation can achieve the lower resolution. Furthermore, if a user is able to decode both the base bits and the refinement bits, a higher resolution is achieved. The enhancement layer cannot reconstruct a higher resolution alone. It has to reuse the information of the lower resolution embedded in the base layer. In order to provide two different resolutions using a nonscalable codec, the media must be encoded twice and the media packets for different resolutions cannot reuse the information from each other. Since the base layer packets encoded by a scalable codec can be reused by the enhancement layer packets, the scalable codec is more efficient than the nonscalable codec in providing multiresolution media simultaneously. In this case, the source packets contributing to the low resolution are allocated to the base bits of the hierarchical constellation and the packets which only contribute to the high resolution are carried by the refinement bits. The users close to the station are able to get all packets decoded and receive a high resolution program. Due to reduced radio signal attenuation, the users far away from the station will probably not be able to decode the refinement bits, but they can still decode the packets for low resolution with acceptable quality.

Note however that the flexibility introduced by hierarchical modulation does not come without a price. In [16], Jiang and Wilford illustrated that a penalty of slightly reduced SNR in base layer bits is introduced by hierarchical modulation. This penalty has equal impact on both scalable and nonscalable codec in a hierarchical system.

However, as we shall see in Section 2, hierarchical modulation also imposes a second performance penalty, namely, for a given choice of hierarchial constellation, and fixed target bit error rates of the two streams, the system will almost certainly be operating at a higher overall SNR than is needed to satisfy the target BERs.

In this paper, we will show how the constellation can be dynamically adapted at the physically layer in order to remove this performance penalty. This adaptation can be 
done at a session level, or even with finer granularity (e.g., at a one-second interval) in response to the changing dynamics of the transmitted bit-streams.

The paper is presented as follow. In Section 2 we discuss the basic analytical tools for calculating bit error rates in a sample hierarchical system. A simulation of the single-user scenario, a simulation of the multiuser scenario and their results are described in Sections 3 and 4, separately. Section 5 concludes this paper.

\section{Error Rate Analysis and Optimization in Hierarchical Modulation}

As introduced above, hierarchical modulation is a physical layer modulation technique in which the received signal constellations can be treated in two (or more) parts, by first making coarse decisions about the constellation location, followed by a refined decision on the exact location. Figure 1 shows a 16-QAM constellation diagram to illustrate hierarchical modulation. The data carried by this constellation is broken into two classes: a low priority (LP) and high priority (HP) class. The bits from the HP stream are used to select the quadrant of the constellation point, and the LP stream is used to choose the exact constellation point. The notations $d_{1}$ and $d_{2}$ represent the intra- and interconstellation group distances, respectively. The ratio $k=$ $d_{1}^{\prime} / d_{2}$ is an important parameter, as it defines the achievable error rates of the system in the presence of noise. When $k$ is equal to 1 , the constellation reverts to a standard 16-QAM constellation. When $k$ is larger than 1 , the HP stream is more heavily protected against noise than the LP stream. This is compatible with the typical definition of constellation ratio in DVB-T/DVB-H standard [13].

Before assigning the HP and LP streams to Hierarchical Modulation constellation points, we can decrease the bit error probability of the streams by using standard coding techniques such as convolutional coding. A high-rate code is suitable for the LP bit stream because of its lower bit error rate demand. Using different rates of code in the HP and LP bit streams is helpful in achieving arbitrary target bit error rates in the Physical Layer.

Exact (in $M$ ) BER expressions for uniform $M$-QAM over an additive white Gaussian noise (AWGN) channel have been developed in $[17,18]$ based on signal-space concepts and a recursive algorithm, respectively. Exact expressions for the BER of 16-QAM and 64-QAM in nonfading and frequency flat fading channels were derived in [19]. The exact and generic (in $M$ ) expression for the BER of uniform square QAM in the presence of AWGN channel was obtained in [20].

For uncoded hierarchical constellation scenarios, an approximate BER expression is described in $[9,10]$ for 4/16-QAM, 4/64-QAM and in [10] for multicast $M$-PSK. Reference [21] obtains exact and generic expressions in $M$ for the BER of the 4/M-QAM (square and rectangular) constellations over additive white Gaussian noise (AWGN) and fading channels. Over the AWGN channel, these expressions can be described by a weighted sum of complementary error functions.

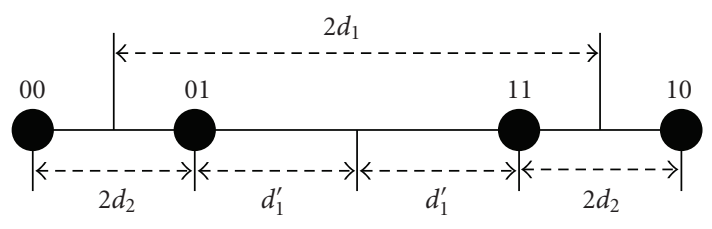

FIgURE 2: 4-PAM constellation.

In the analysis and simulations which follow, we assume two bit streams, separately fed into convolutional encoders with code rates $R_{1}$ and $R_{2}$, which are then gray-coded and modulated onto a 16-QAM constellation. After the encoding and modulation, the two streams are converged into one symbol sequence and transmitted through an AWGN channel. In the receiver the symbols contaminated by noise are demodulated using a Maximum-LikelihoodSequence-Estimation technique (Viterbi).

In order to determine the performance of this hierarchical modulated scheme, we carry out an analysis of the error probability for the uncoded case. An exact bit error probability expression has been derived in [21]. In this section, the expression will be further developed into a simpler form. This will allow us to minimize the overall SNR which satisfies the target BERs. For the sake of clarity, we will start the analysis from the original step.

As described in [22], the 16-QAM constellation is equivalent to two 4 PAM signals on quadrature carriers. Since the signals in the phase-quadrature components can be perfectly separated at the demodulator, the probability of error for QAM can be easily determined from the probability of error for PAM. Therefore, the probability of a bit error for the $M$-ary QAM is

$$
P_{M}=\frac{1}{2}\left(P_{i, \sqrt{M}}+P_{q, \sqrt{M}}\right)
$$

where $P_{i, \sqrt{M}}$ and $P_{q, \sqrt{M}}$ are the error probabilities of the $\sqrt{M}$-ary PAMs with one-half the average power in each quadrature signal of the equivalent QAM system. It should be emphasized here that the error probability discussed here is bit error, which is different from the symbol error in [22].

The signal points for the unevenly spaced gray-encoded 4-PAM constellation are described in Figure 2.

The error probability for the bits contained in the HP stream is

$$
P_{H}=\frac{1}{2}\left[\frac{1}{2} P\left(\left|r-s_{m}\right|>d_{1}-d_{2}\right)+\frac{1}{2} P\left(\left|r-s_{m}\right|>d_{1}+d_{2}\right)\right],
$$

where $r$ is the received symbol contaminated by white Gaussian noise with zero-mean and variance $\sigma_{n}^{2}=(1 / 2) N_{0}$, and $s_{m}$ is the transmitted symbol (i.e., $r=s_{m}+n$ ). We assume 
that each symbol is equiprobable. Given this AWGN channel, the error probability can be given generically as

$$
\begin{aligned}
P & =\frac{1}{2} P\left(\left|r-s_{m}\right|>d\right) \\
& =\frac{1}{2} \frac{2}{\sqrt{\pi N_{0}}} \int_{d}^{\infty} e^{-x^{2} / N_{0}} d x \\
& =Q\left(\sqrt{\frac{2 d^{2}}{N_{0}}}\right) .
\end{aligned}
$$

The average bit energy is

$$
\varepsilon_{b}=d_{1}^{2}\left(1+\frac{1}{(k+1)^{2}}\right)=d_{2}^{2}\left[(k+1)^{2}+1\right],
$$

where $k=d_{1}^{\prime} / d_{2}$ and $d_{1}=d_{2}+d_{1}^{\prime}$. Let

$$
\begin{aligned}
& A=(1+k)^{2}, \\
& B=A+1 .
\end{aligned}
$$

Using (1)-(5), we obtain the error probability for the HP bit of 4-PAM as

$$
P_{H}=\frac{1}{2}\left[Q\left(\sqrt{\frac{2 \varepsilon_{b}(k+2)^{2}}{B N_{0}}}\right)+Q\left(\sqrt{\frac{2 \varepsilon_{b} k^{2}}{B N_{0}}}\right)\right] .
$$

From the same argument, we can determine the error probability for the LP bit of the 4-PAM constellation as

$$
\begin{aligned}
P_{L}= & Q\left(\sqrt{\frac{2 \varepsilon_{b}}{B N_{0}}}\right)+\frac{1}{2} Q\left(\sqrt{\frac{4 \varepsilon_{b}\left(2 k^{2}+5 k+4\right)}{B N_{0}}}\right) \\
& -\frac{1}{2} Q\left(\sqrt{\frac{4 \varepsilon_{b}\left(2 k^{2}-5 k+4\right)}{A N_{0}}}\right) .
\end{aligned}
$$

Assume that the distances between the corresponding signal points in the Imaginary component and the Quadrature component are same:

$$
P_{i, \sqrt{M}}=P_{q, \sqrt{M}} .
$$

By substituting the error probabilities for the PAMsystem, we can obtain the corresponding QAM-system BERs as a function of $k$ :

$$
\begin{aligned}
P_{\mathrm{HM}}= & \frac{1}{2}\left[Q\left(\sqrt{\frac{2 \varepsilon_{b}(k+2)^{2}}{B N_{0}}}\right)+Q\left(\sqrt{\frac{2 \varepsilon_{b} k^{2}}{B N_{0}}}\right)\right], \\
P_{\mathrm{LM}}= & Q\left(\sqrt{\frac{2 \varepsilon_{b}}{B N_{0}}}\right)+\frac{1}{2} Q\left(\sqrt{\frac{4 \varepsilon_{b}\left(2 k^{2}+5 k+4\right)}{B N_{0}}}\right) \\
& -\frac{1}{2} Q\left(\sqrt{\frac{4 \varepsilon_{b}\left(2 k^{2}-5 k+4\right)}{A N_{0}}}\right) .
\end{aligned}
$$

Figure 3 uses the expressions derived above to calculate the BER rates for the LP and HP streams using a fixed 16QAM constellation with $k=1$, and typical convolutional

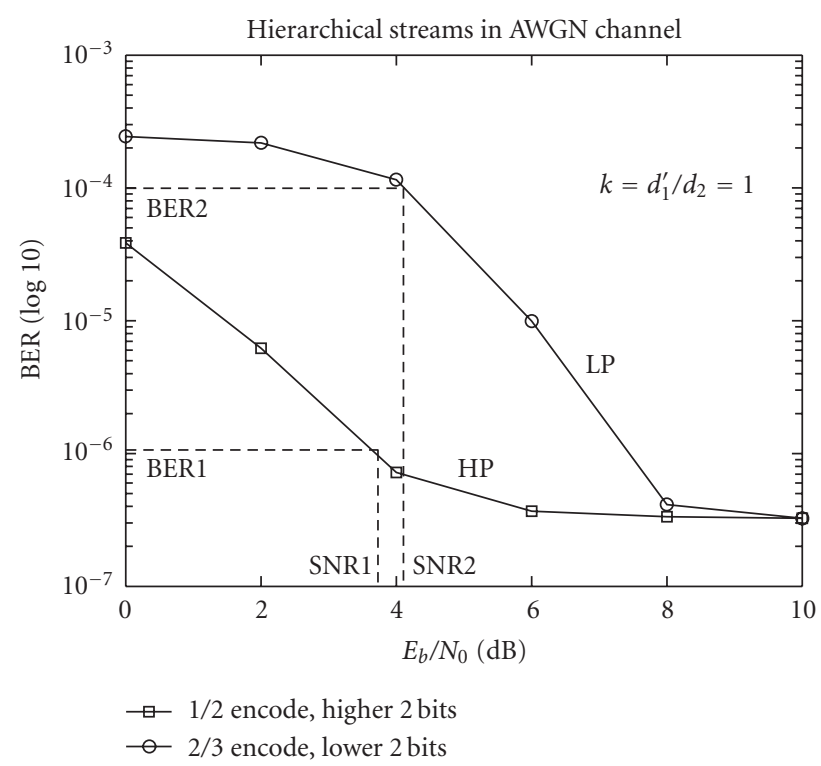

FIgure 3: Bit error rate curves for a convolutional coded hierarchical modulation scheme, with a fixed value of $k=1$.

codes used on both streams. In this example, the target BER is chosen as $1 e-6$ for the HP data and $1 e-4$ for the LP stream. It illustrates the potential penalty of operating a fixed hierarchical modulation scheme. In this case, at an SNR of 4.1, we satisfy the LP BER, but we actually exceed the target BER for the HP bit. In a sense, we are therefore transmitting more signal power than is necessary to meet the system requirements.

\subsection{Optimization of Hierarchical Modulation for AWGN} Channel. From (9) we can derive the Signal-to-Noise Ratio (SNR) for low priority bits and high priority bits as a function of space ratio $k$ and the target bit error rate for high priority bits and low priority bits:

$$
\begin{aligned}
& \left(\frac{\varepsilon_{b}}{N_{0}}\right)_{\mathrm{HM}}=f_{\mathrm{HM}}\left(P_{\mathrm{HM}}, k\right), \\
& \left(\frac{\varepsilon_{b}}{N_{0}}\right)_{\mathrm{LM}}=f_{\mathrm{LM}}\left(P_{\mathrm{LM}}, k\right) .
\end{aligned}
$$

The overall SNR required by the transmission of both high priority bits and low priority bits is the bigger one of the SNR described by (10). Thus, given target bit error rates for high priority bits and low priority bits,

$$
\begin{aligned}
P_{\mathrm{HM}} & =\mathrm{BER}_{\mathrm{HM}}, \\
P_{\mathrm{LM}} & =\mathrm{BER}_{\mathrm{LM}},
\end{aligned}
$$

the optimization of the hierarchical modulation can be described by the following equation:

$$
\min _{k \in R, k>0}\left(\max \left(\left(\frac{\varepsilon_{b}}{N_{0}}\right)_{\mathrm{HM}},\left(\frac{\varepsilon_{b}}{N_{0}}\right)_{\mathrm{LM}}\right)\right) .
$$

Since the $Q$ function in (9) does not have an expression with finite number of coefficients, it is difficult to get an exact 


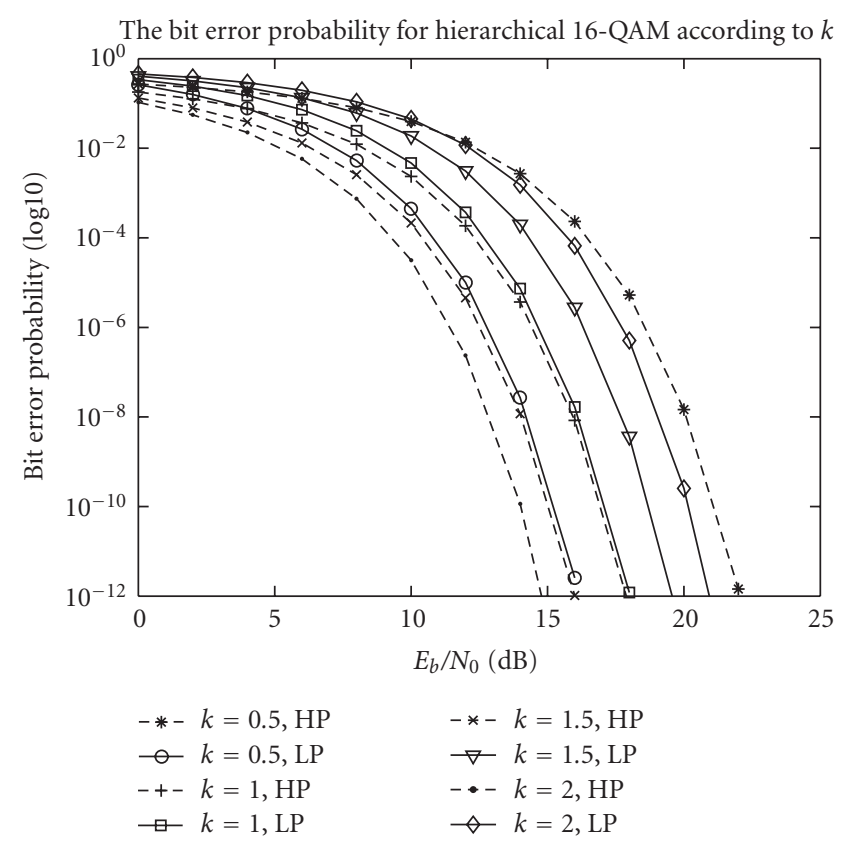

FIGURE 4: Bit error probability versus SNR per bit for various $k$ values.

expression for (10). There are several approximations proposed in [23-26]. However, all these approximations are suitable for a specific range of the independent variable. For example, when the independent variable $x$ is smaller and far away from $1(x \ll 1)$, an approximation of $Q$ function is derived from the Maclaurin series:

$$
Q(x)=\frac{1}{2 \pi}\left(x-\frac{1}{6} x^{3}+\frac{1}{40} x^{5}-\frac{1}{336} x^{7}+\frac{1}{3456} x^{9}+\cdots\right) .
$$

The objective of the optimization is to find out an optimum number $k$ given $(k>0, k \in R)$, which leads to a minimum overall SNR. Thus the above approximation of $Q$ function is not suitable. In this section, we first analyze the property of (10) by aid of the BER versus SNR curve. Then, a realistic method is used to calculate the tabulation of the overall SNR versus the space ratio $k$ and the target BER for high priority bits and low priority bits.

Figure 4 is drawn according to (9). It gives the BER curves versus global $E_{b} / N_{0}$ for various values of $k$. It illustrates along with the increment of $k$, the BER curve for HP bits moves backward while the BER curve for LP bits moves forward along the SNR axis. That is, for a fixed BER value, the SNR for HP bits monotonically decreases as $k$ increases, and the SNR for LP bits monotonically increases in response to $k$. As $k$ increases, the SNR for HP and LP bits cross; thus, for any target bit error rate, the gap between SNR for HP bit and the SNR for LP bits equals zero for some value of $k$. Because the overall SNR is the bigger on of the SNR for high priority bits and the SNR for low priority bits, we conclude that we get the minimum overall SNR when the SNR for HP is equal to the SNR for LP which meets the target BER.

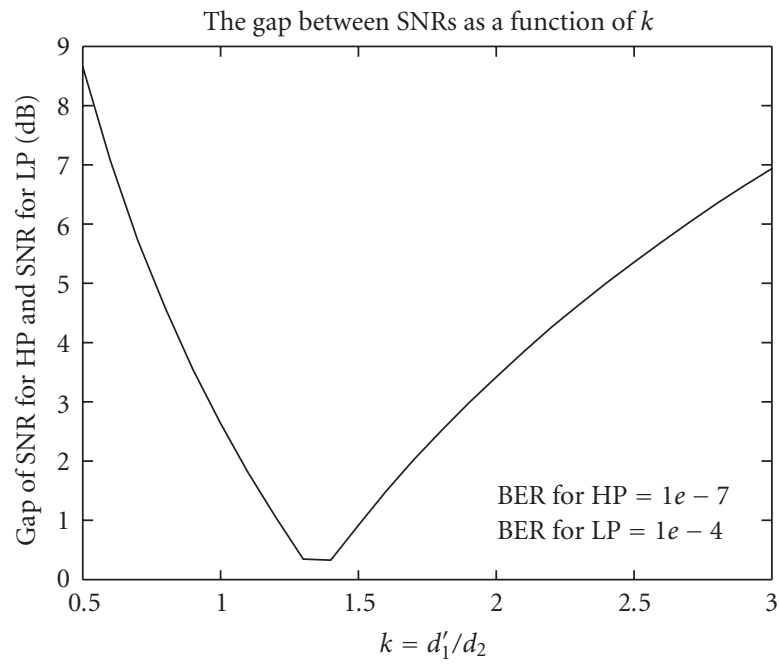

FIGURE 5: SNR Gap versus $k$ curve.

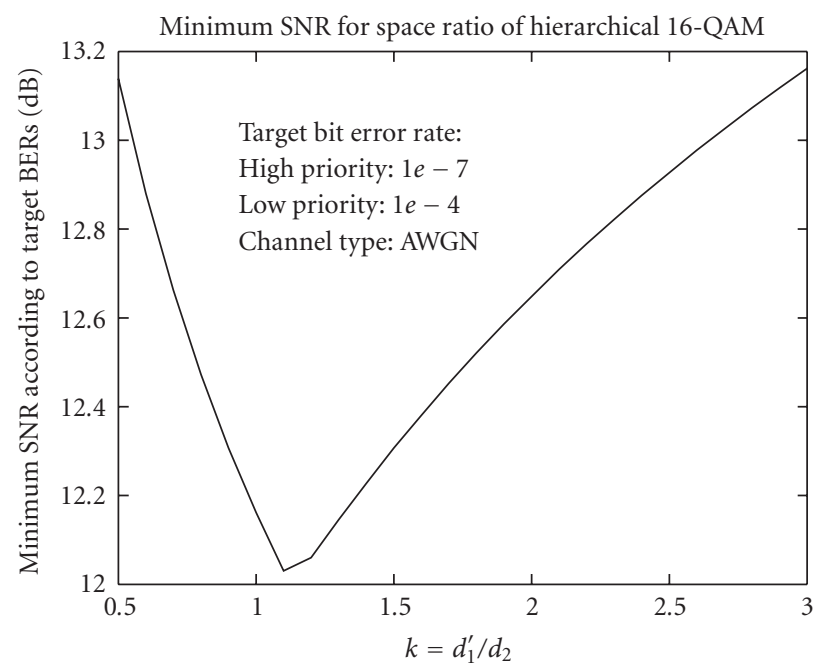

FIGURE 6: SNR versus $k$ curve.

Given the BER formulae in (9), we can easily estimate the required $E_{b} / N_{0}$ to meet the target Bit Error Rates. Through tracking the gap between these SNRs per bit in response to $k$, we can find the $k$ corresponding to the minimal gap. An example is shown in Figures 5 and 6, where the target BERs are $1 \times 10^{-7}$ and $1 \times 10^{-4}$. Figure 5 shows that the $k$ which produces a zero-gap is about 1.4, and Figure 6 shows that the corresponding SNR per bit is approximately $11.4 \mathrm{~dB}$.

Figure 7 shows the comparison of the required SNR per bit for $k=1$ (normal 16-QAM) and $k=1.4$ (Optimized Hierarchical Modulation) in order to achieve the desired BERs, and shows that about $2 \mathrm{~dB}$ savings can be achieved by optimization.

2.2. Optimization of Hierarchical Modulation for Flat Rayleigh Fading Channel. Since an OFDM system is employed in the simulation, the multipath Rayleigh fading channel is converted to a flat Rayleigh fading channel for a specific 


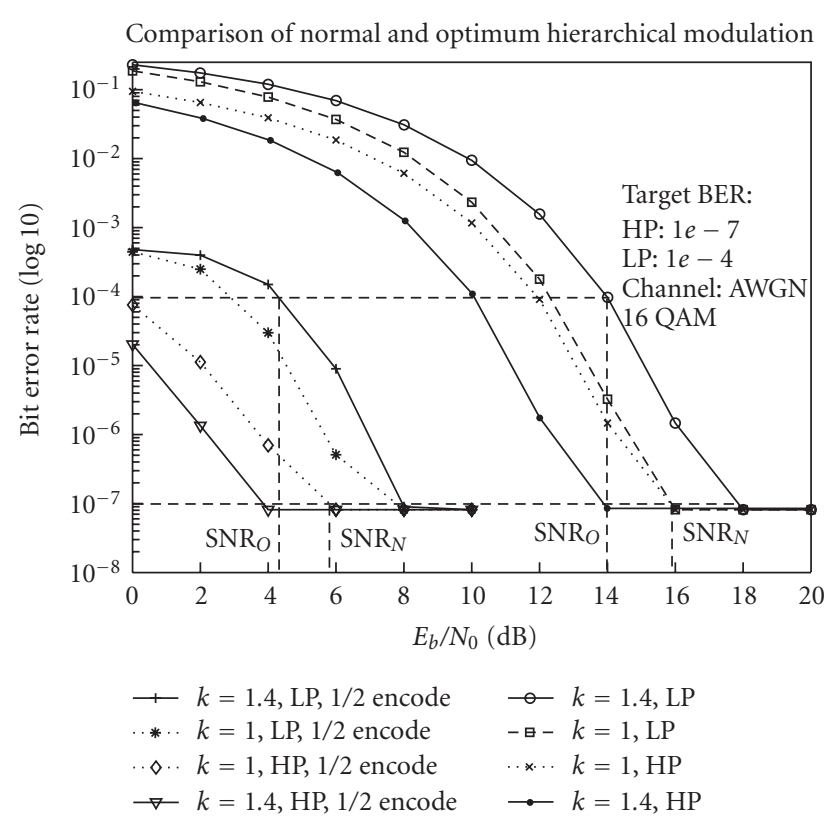

FIgURE 7: Comparison of required SNR per bit for $k=1$ and $k=$ 1.4 .

subcarrier, given that the cyclic prefix length is longer than the number of taps used by the multipath fading channel. In this section, the bit error probability of high priority bits and low priority bits over flat Rayleigh fading channel are deployed and the optimization of the hierarchical modulation over flat Rayleigh fading channel is explained.

In the simulation of this paper we employed a frequencyselective fading channel. That is, we simulated an indoor small scale multiple reflective paths radio environment and there is no line-of-sight component. There is relatively slow motion between the transmitter and the receiver. The mathematical model of the multipath radio channel is expressed by (14):

$$
H\left(n T_{s}\right)=\sum_{k=0}^{N-1} a_{k} h\left(n T_{s}-k T_{s}\right) .
$$

In the equation above, $T_{s}$ denotes the sample period and $h\left(n T_{s}-k T_{s}\right)$ simulates multipath delay components of the fading channel. The coefficient $a_{k}$ represents the attenuation of the $k$ th path. Each $h\left(n T_{s}-k T_{s}\right)$ can be modeled by

$$
h_{n}(t)=x_{n}(t)+j y_{n}(t),
$$

in which the $x_{n}(t)$ and $y_{n}(t)$ are independent and identical distributed (i.i.d.) Gaussian random variable with mean $\mu=$ 0 and variance $\sigma^{2}$. The magnitude $\left|h_{n}(t)\right|$ has Rayleigh power density function (PDF) described by

$$
p(r)=\frac{r}{\sigma^{2}} e^{-r^{2} / 2 \sigma^{2}}, \quad r \geq 0 .
$$

In one subcarrier of the OFDM symbol, the multipath Rayleigh Fading channel is converted to a single path channel:

$$
H\left(n T_{s}\right)=a h\left(n T_{s}\right)
$$

or in normalized continuous version,

$$
H(t)=h(t)
$$

The system channel model is described by

$$
y(t)=h(t) x(t)+n
$$

in which $y(t)$ is the received signal, $x(t)$ is the transmitted signal, $h(t)$ is the complex flat Rayleigh fading component and $\mathrm{n}$ is Additive White Gaussian Noise (AWGN) with mean 0 and variance $\sigma_{0}^{2}$. When the received signal is equalized in the receiver, the flat Rayleigh fading component is estimated by the receiver and used to divide (19). The following equation is derived from (19):

$$
\hat{y}(t)=x(t)+\frac{n}{h(t)}
$$

Equation (20) indicates that by taking into account the flat Rayleigh Fading component $h(t)$ the generic error probability over AWGN channel as described by (3) becomes

$$
P=Q\left(\sqrt{\frac{|h|^{2} 2 d^{2}}{N_{0}}}\right),
$$

in which $|h|$ is a Rayleigh distribution random variable and $|h|^{2}$ is chi-square random distributed with two degrees of freedom, if the variance of $\operatorname{Re}(h)$ and $\operatorname{Im}(h)$ is 1 , which is an assumption without loss of generality. Thus, the following equation is used to calculate the generic error probability over flat Rayleigh fading channel:

$$
P_{h}=\int_{0}^{\infty} \frac{1}{2} \operatorname{erfc}(\sqrt{\gamma}) p(\gamma) d \gamma
$$

in which $\gamma=|h|^{2} d^{2} / N_{0}$ and $\operatorname{erfc}(x)$ is called complementary error function. Complementary function has the following relation with $Q$ function:

$$
\operatorname{erfc}(x)=\frac{2}{\sqrt{\pi}} \int_{x}^{\infty} 2^{-t^{2}} d t=2 Q(2 x)
$$

The PDF for chi-square distributed random variable with two degrees of freedom is described by

$$
p(\gamma)=\frac{1}{2 d^{2} / N_{0}} e^{-\gamma /\left(2 d^{2} / N_{0}\right)}
$$

By introduction of (24) to (22) the generic bit error probability over flat Rayleigh fading channel is derived as

$$
P_{h}=\frac{1}{2}\left(1-\sqrt{\frac{2 b^{2} / N_{0}}{2 b^{2} / N_{0}+1}}\right) .
$$


From (25), (1), (4), (5), and (6), we can derive the bit error probability of high priority bits and low priority bits for Hierarchical Modulation over flat Rayleigh fading channel:

$$
\begin{aligned}
P_{\mathrm{HM}, h}= & \frac{1}{2}-\frac{1}{4} \sqrt{\frac{2 \varepsilon_{b}(k+2)^{2} / B N_{0}}{2 \varepsilon_{b}(k+2)^{2} / B N_{0}+1}} \\
& -\frac{1}{4} \sqrt{\frac{2 \varepsilon_{b} k^{2} / B N_{0}}{2 \varepsilon_{b} k^{2} / B N_{0}+1}}, \\
P_{\mathrm{LM}, h}= & \frac{1}{2}-\frac{1}{2} \sqrt{\frac{2 \varepsilon_{b} / B N_{0}}{2 \varepsilon_{b} / B N_{0}+1}} \\
& -\frac{1}{4} \sqrt{\frac{4 \varepsilon_{b}\left(2 k^{2}+5 k+4\right) / B N_{0}}{4 \varepsilon_{b}\left(2 k^{2}+5 k+4\right) / B N_{0}+1}} \\
& +\frac{1}{4} \sqrt{\frac{4 \varepsilon_{b}\left(2 k^{2}-5 k+4\right) / A N_{0}}{4 \varepsilon_{b}\left(2 k^{2}-5 k+4\right) / A N_{0}+1}} .
\end{aligned}
$$

From (26) we can derive the Signal-to-Noise Ratio (SNR) for low priority bits and high priority bits as a function of space ratio $k$ and the target bit error rate for high priority bits and low priority bits over flat Rayleigh fading channel:

$$
\begin{aligned}
& \left(\frac{\varepsilon_{b}}{N_{0}}\right)_{\mathrm{HM}, h}=f_{\mathrm{HM}, h}\left(P_{\mathrm{HM}}, k\right), \\
& \left(\frac{\varepsilon_{b}}{N_{0}}\right)_{\mathrm{LM}, h}=f_{\mathrm{LM}, h}\left(P_{\mathrm{LM}}, k\right) .
\end{aligned}
$$

The optimization of the hierarchical modulation over flat Rayleigh fading channel can be described by the following equation:

$$
\min _{k \in R, k>0}\left(\max \left(\left(\frac{\varepsilon_{b}}{N_{0}}\right)_{\mathrm{HM}, h},\left(\frac{\varepsilon_{b}}{N_{0}}\right)_{\mathrm{LM}, h}\right)\right) .
$$

Similar to the situation described in Section 2.1, it is difficult to get an exact expression for (27). We can employ a realistic method to calculate the tabulation of the overall SNR versus space ratio $k$. On the other hand, we can analyze the feature of the relation between overall SNR and space ratio $k$ by drawing the flat Rayleigh fading version of Figures 5 and 6. Figure 8 shows the gap between the required SNR for high priority bits and the required SNR for low priority bits, in order to meet the target average BER for high priority bits and low priority bits over flat Rayleigh fading channel. Figure 9 shows the local minimum overall SNR required for the target average BER versus space ratio $k$ over flat Rayleigh fading channel. In the given example, the target average BERs for HP and LP are $10^{-7}$ and $10^{-4}$. The local optimum $k$ value is approximately $k_{\min }=37.07$. The corresponding local minimum SNR is $\left(E_{b} / N_{0}\right)_{\min }=17.72 \mathrm{~dB}$. In practice, when the space ratio $k \gg 3$, it implies that the hierarchical 16QAM constellation is degraded into a 4-QAM constellation. This means, in a flat Rayleigh fading channel, the low priority bits of a 16-QAM hierarchical constellation is very easy to be distorted and sensitive to channel noise. In order to conquer the flat fading distortion, a convolutional channel coding method is employed in the simulation. The impact of channel coding is discussed in the following sections.

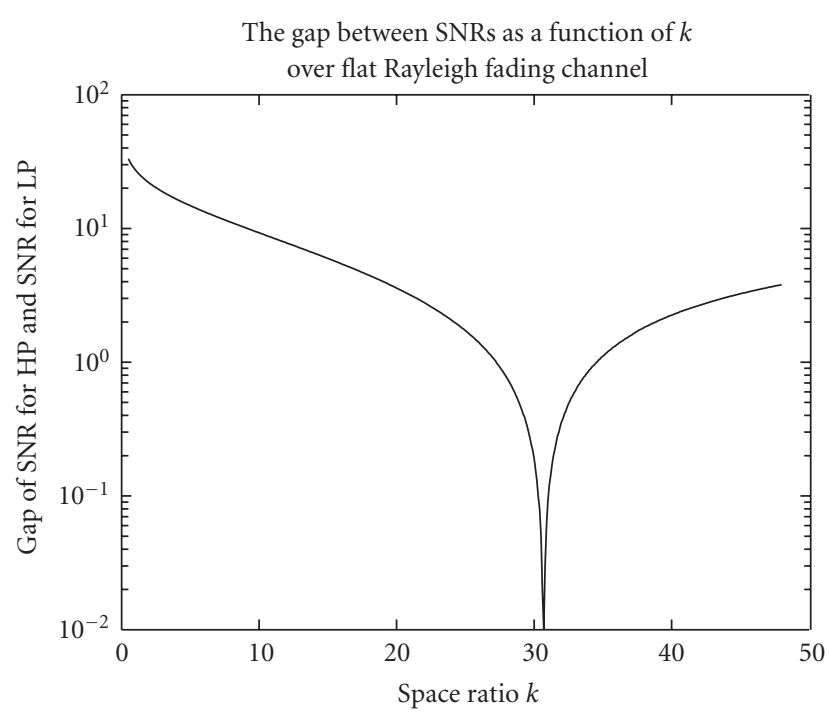

FIGURE 8: SNR Gap versus $k$ curve over flat Rayleigh fading channel. The target ber for high priority bits is $1 e-7$ and the target ber for low priority bits is $1 e-4$.

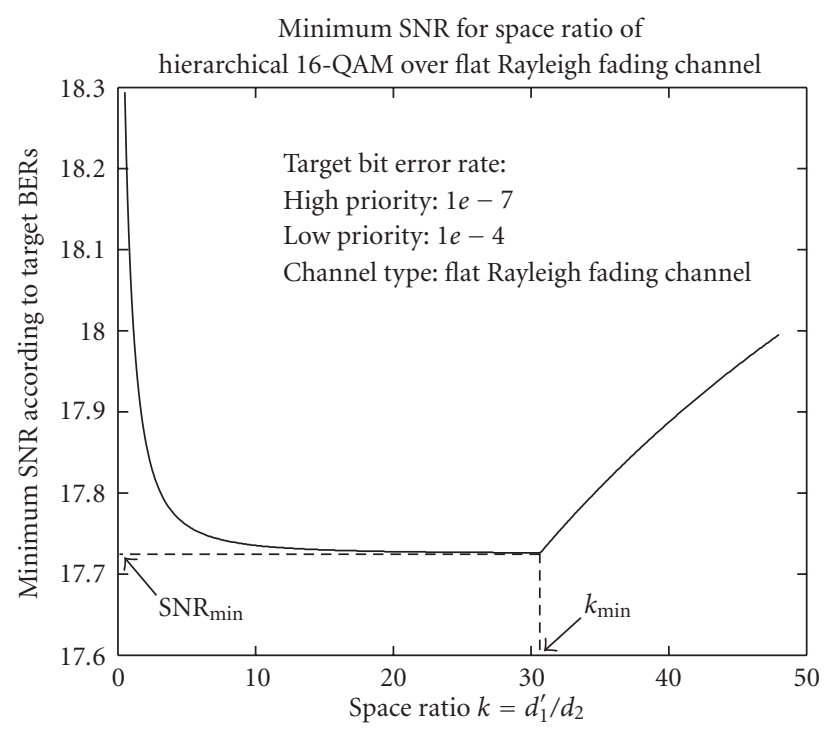

FIGURE 9: SNR versus $k$ curve over flat Rayleigh fading channel. The overall SNR gets minimum value of $17.72 \mathrm{~dB}$ when the space ratio $k=37.07$.

2.3. Analysis of Packet Error Rate over AWGN Channel. The previous analysis is based on bit error rate. In practice, higher layers may be packet-oriented, so that package error rate is the more important parameter. We can make a simple mapping from BER to expected PER, under some simple assumptions. Assuming that the probability of decoding one bit wrongly $\left(P_{b}\right)$ is a stationary uncorrelated process, we can consider the decoded bit stream as a Poisson process. This yields the relationship between BER and PER:

$$
P_{p}=1-e^{-P_{b} L},
$$

in which, $P_{p}$ is PER, $P_{b}$ is BER and $L$ is the package length. 


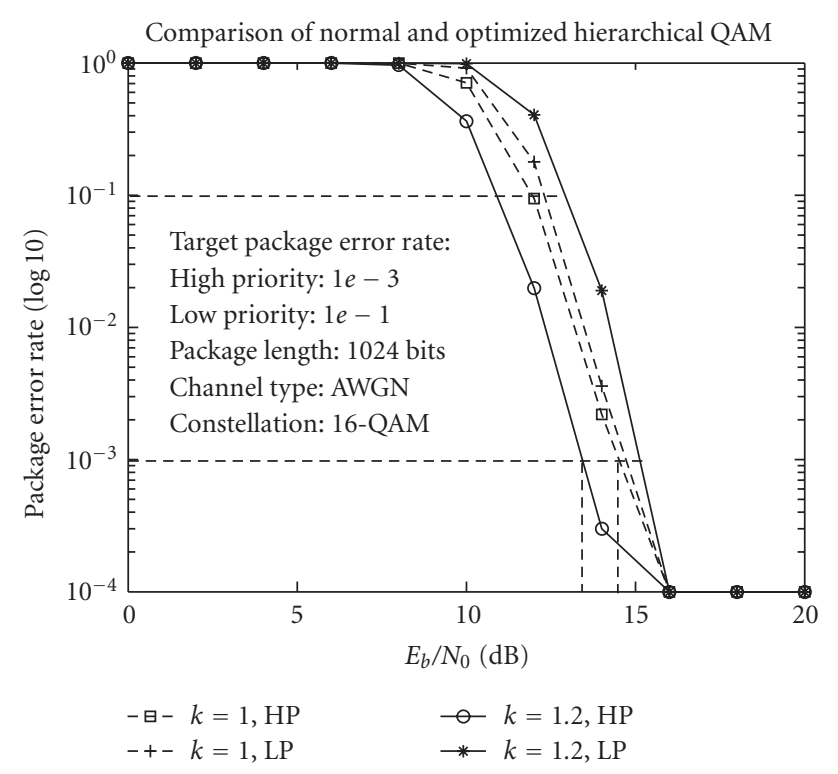

FIgURe 10: Package error rate versus SNR per bit.

Using (9)-(29), we find that for a fixed $k$, the required SNR will increase in response to increased packet length.

The packet length is affected by the tradeoff between source coding efficiency and packet error rate. Given a fixed packet length, we can achieve the corresponding optimum space ratio $k$.

Figure 10 shows a comparison of SNR per bit for $k=$ 1 (Normal 16-QAM) and $k=1.2$ (optimized Hierarchical Modulation) for a desired package error rate. In this case, about $1 \mathrm{~dB}$ power saving is achieved by optimization.

2.4. Impact of Coding on Performance. Analytical results to date have been based on uncoded bit error rates. In practice, the performance of coded hierarchical modulation systems is of more practical interest. The effect of coding will shift the BER curve to the left by the coding gain.

For the HP and LP streams, the two BER curves will in general be shifted by different amounts (since coding gain is a function of the code, and the SNR). However, the coding gain is fixed for a given code rate and SNR, given fixed target BERs. Thus, we can apply a known correction factor to the optimum space ratio $k$ in the case of encoded bits. For example, Table 1 gives the coding gain difference between the shifts of HP and LP BER curves for the case where a rate $R=1 / 2$ is used in both streams. Hence, we can iteratively determine the optimum space ratio in the case of rate $1 / 2$ encoders and 16-QAM Hierarchical modulations.

Figure 11 shows the coding gain of $1 / 2$ convolutional coded hierarchical method for specific targe bit error rate for high periority and low priority stream.

\section{Single-User Scenario Simulation and Results}

As a proof-of-concept of the use of the Optimum Hierarchical Modulation scheme for single user in scalable video

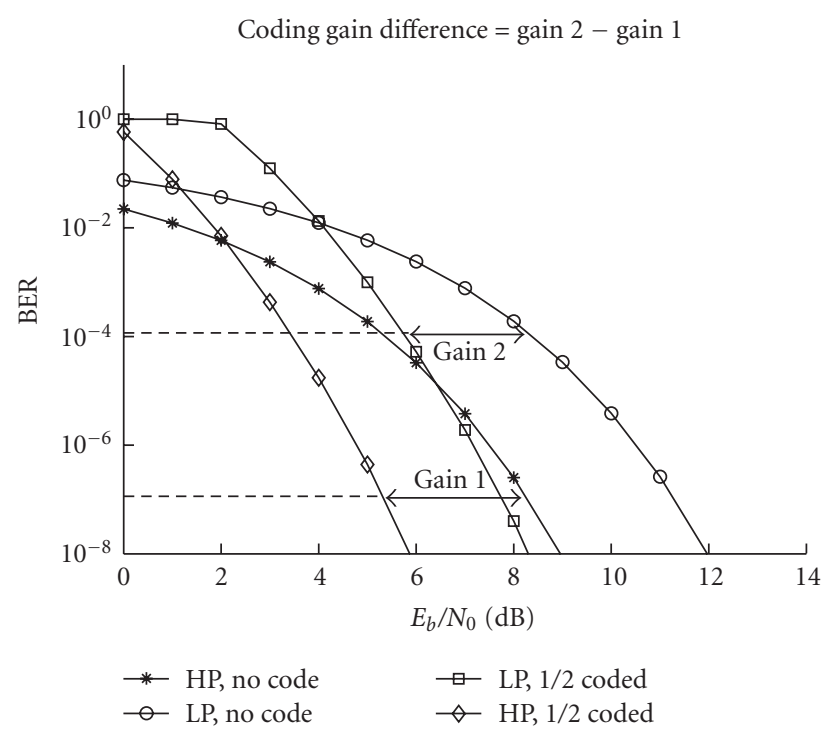

FIgURE 11: Comparison of coded and uncoded BERs versus SNR per bit (16-QAM modulated).

TABLE 1: Coding gain of BER curve according to space ratio $k$ at BER of $10^{-4}$ and $10^{-7}$.

\begin{tabular}{lcccccc}
\hline$k\left(d_{1}^{\prime} / d_{2}\right)$ & 0.5 & 1 & 1.5 & 2 & 2.5 & 3 \\
\hline $\begin{array}{l}\text { Difference in } \\
\text { Coding Gain }\end{array}$ & 8.3 & 2.5 & -1.1 & -3.6 & -5.6 & -6.9 \\
$(\mathrm{~dB})$ & & & & & & \\
\hline
\end{tabular}

delivery, we send a still image through an AWGN single carrier channel.

The convolutional code of rate $1 / 2$ and 16-QAM Hierarchical Modulation is employed for transmission. The data bits with higher priority and lower priority are convolutional coded and padded with parity bits. The coded bits with high priority are used to select the base bits in 16-QAM constellation and the coded bits with low priority are used to select the refinement bits in the constellation. The average distances of the base bits and the refinement bits can be tuned in order to give an optimized overall image quality (all save the $E_{b} / N_{0}$ under the same quality).

We employ a specific example of a scalable still image encoder. The $64 \times 64$ pixels image is processed by a progressive encoder called the Embedded Zero-tree WaveletSpatial Orientation Tree (EZW-SOT) [27]. An embedded code represents a sequence of binary decisions that distinguish an image from the all gray image. The embedded coding possesses the property that all the bits are ordered in importance in the bit stream. The importance of the bits can be determined by the precision, magnitude, scale, and spatial location of the wavelet coefficients. For example, there are several real numbers described by 4 digits$a \cdot b c d$. The digit $a$ is the most significant digit of each number and the $d$ is the least significant digit. Thus, the numbers can be stored by a new order in significance, say, $a 1, a 2, \ldots, b 1, b 2, \ldots, c 1, c 2, \ldots, d 1, d 2, \ldots$ Using embedded coding, a decoder can stop decoding at any position and 


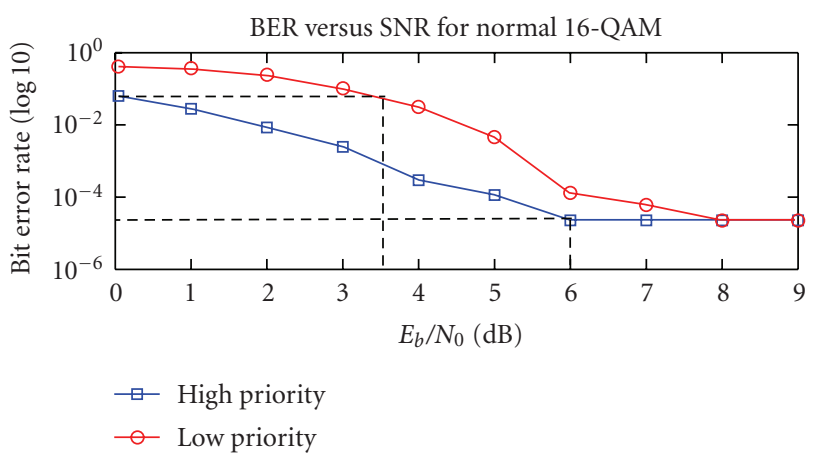

(a)

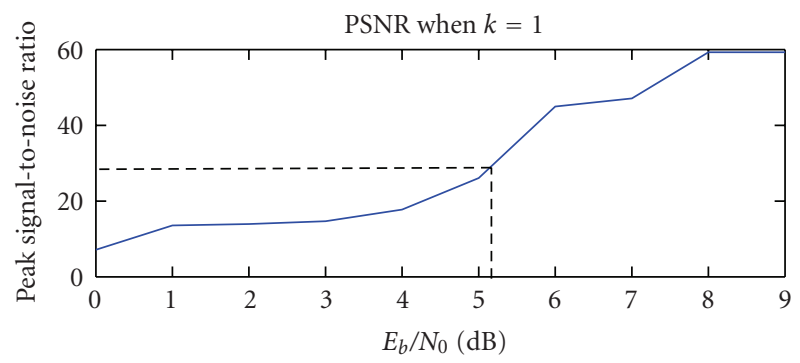

(b)

FIGURE 12: BER and PSNR curve for Hierarchical Modulation of $k=1$.

an optimized quality of the same image will be achieved. A discrete wavelet transform provides a multiresolution presentation of the image. The wavelet coefficients can be embedded coded according to their significance. The zerotree coding provides a binary map, which can indicate the positions of the significant wavelet coefficients.

Since the coded bits are ordered in importance, it is possible to partition the bits in any position arbitrarily. The encoded bit stream is then divided into two-priority classes, with target BERs of $1 \times 10^{-3}$ and $1 \times 10^{-5}$. In a first simulation, we choose a space ratio $k=1$ (conventional 16-QAM modulation). The BER of transmission and PSNR of the resulting decoded image are shown in Figure 12. In the simulations we assume no retransmission of any data. In order to avoid the crash of the decoder, the first data packet which contains the header information for decoding is assumed to be perfectly received. In simulation 2 , we chose the optimum space ratio $k=1.3$ for the coding. The result of simulation 2 is shown in Figure 13. In both cases, $R=1 / 2$ convolutional codes are used for both LP and HP bitstreams. To achieve a desired value of PSNR $=30 \mathrm{~dB}$ (at which it is hard to perceive the quality difference between the decoded image and the original one), simulation 1 has to provide an SNR per bit greater than $5.3 \mathrm{~dB}$, and simulation 2 only needs to provide an SNR per bit of $3.8 \mathrm{~dB}$, so that $1.5 \mathrm{~dB}$ is saved.

\section{Two Users Scenario Simulation and Results}

In the single-user hierarchical modulation scenario, the two or more data channels mapped to the base bits and refinement bits of the constellation points are used to carry

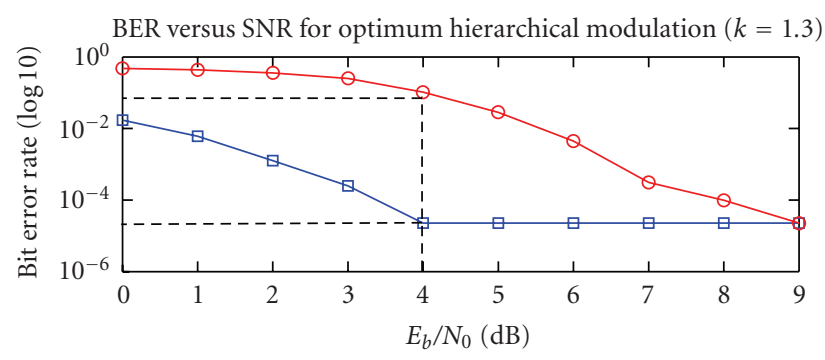

(a)

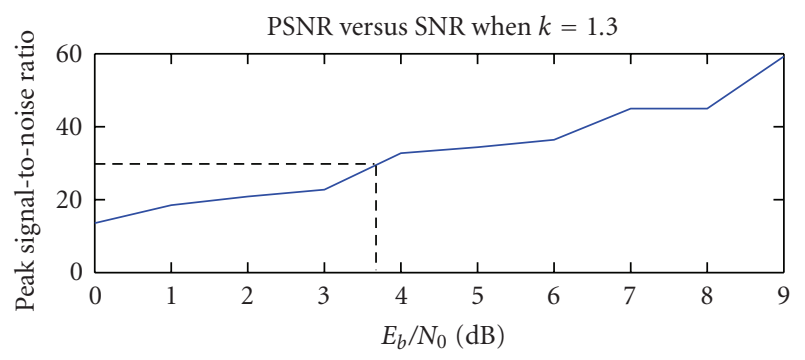

(b)

FIgure 13: BER and PSNR curve for Optimum Hierarchical Modulation of $k=1.3$.

the data belonging to different priority levels of one service aiming at one user. As an alternative to the single user case, in the two users case, the two users are assumed to receive the data carried by the hierarchical constellation points and collect the part useful to them. In our simulation, we transmitted an H.264 scalable coded video trailer in which two different resolution sizes are embedded, a VGA $(640 \times$ 480 pixels $)$ size and a QVGA $(320 \times 240$ pixels $)$ size. The video packets used for decoding the VGA and the QVGA versions are carried by the two different data channels of the hierarchical constellation. All the data packets are encoded using a convolutional code with a rate of $1 / 2$ before being mapped to the constellations. Assuming the video signal is transmitted in an indoor wireless environment, one user is close to the transmitter and has good average $E_{b} / N_{0}$, the other is relatively far from the transmitter and relatively bad average $E_{b} / N_{0}$. The user in a good receiving condition is able to decode most of the data packets and is able to watch the VGA version of the trailer. The user in bad receiving condition cannot obtain enough data packets for decoding a VGA trailer due to the wireless loss, but can decode a QVGA size video with acceptable quality.

According to the scalability in the spatial domain, the video data packets are classified into the base layer packets and the refinement layer packets in resolution. The base layer resolution packets bearing a QVGA sample of the original pictures, that can be used to reconstruct a QVGA size of the original video. The refinement layer packets in resolution carry the refinement information which can be used together with the base layer packets to reconstruct a full VGA sample of the video, as shown in Figure 14.

The bit rate of the refinement layer packets is approximately twice that of the bit rate of the base layer packets. A 4/64-QAM hierarchical constellation is employed, as shown 


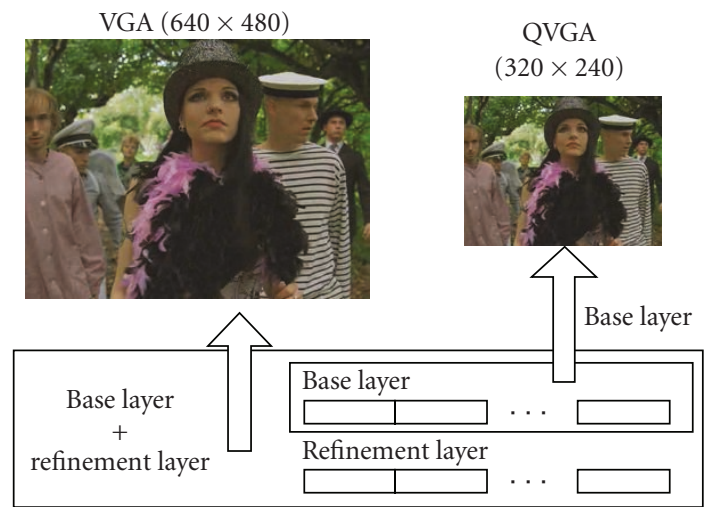

FIgURE 14: The H.264 scalable encoded video composes of two embedded resolutions.

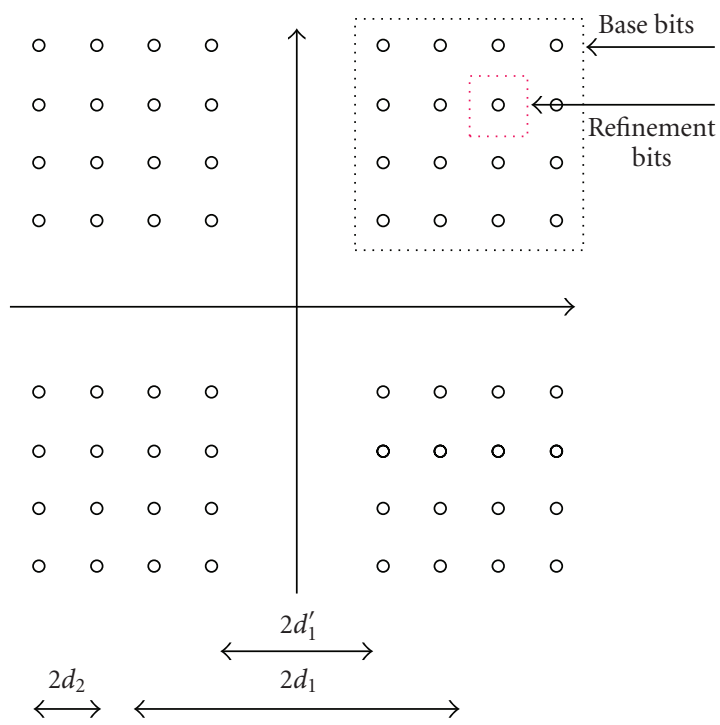

FIGURE 15: The 4/64-QAM hierarchical constellation modulated by two sequences of data bits.

in Figure 15. Each bit from the base layer packets is used to select the four quadrants of a 4/16-QAM constellation and each two bits from the refinement layer packets are used to choose one of the constellation points inside the quadrant selected by the base bit. The $2 d_{1}$ and $2 d_{2}$ represent the intra- and interconstellation group distances, respectively. The ratio of $d_{1}^{\prime}$ and $d_{2}, k=d_{1}^{\prime} / d_{2}$ can be tuned to change the BER performance of the base bits and the refinement bits.

Figure 16 shows the PSNR performance of the VGA version of the H.264 scalable video decoded by the "good condition" user with the different values of the space ratio $k=1,2,4$. We employ slow multipath fading channel for the simulations. The fading channel is modeled by the sum of a series of delayed taps, with each tap is generated by a Rayleigh process. The coefficients of each delayed tap are calculated according to the model A in [28]. The PSNR performance of the QVGA version of the H.264 scalable video decoded by the "bad condition" user is shown in Figure 17. When $E_{b} / N_{0}$ is below $25 \mathrm{~dB}$, increasing the space

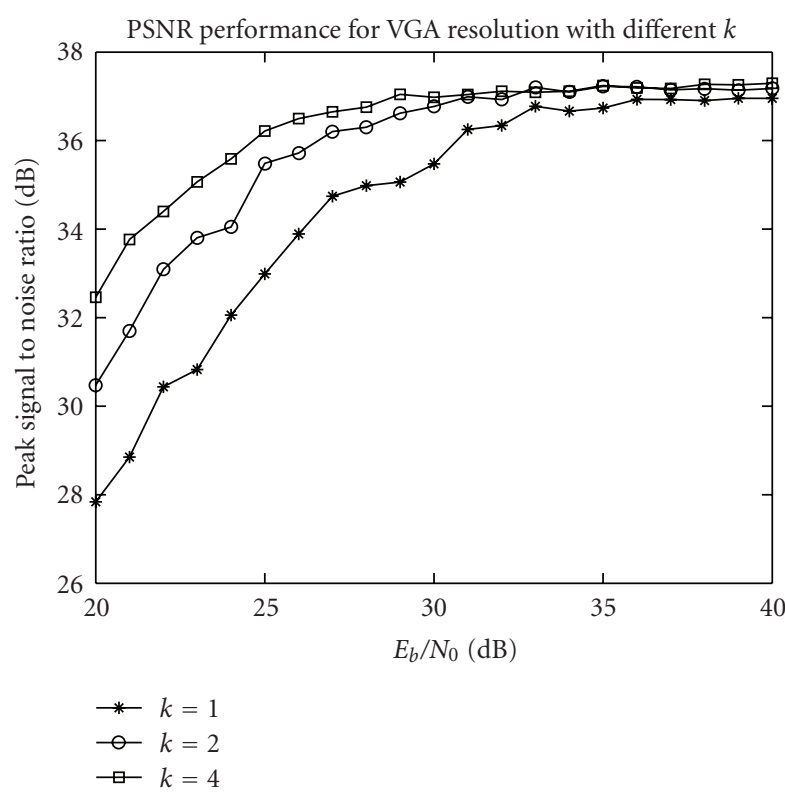

FiguRE 16: The PSNR performance for VGA resolution with different space ratio $k$. The priority of each data packet is labeled and aware of by the MAC layer.

ratio $k$ can improve the PSNR performance significantly for both VGA and QVGA versions. This can be explained because a bigger $k$ means more protection for the high priority level data channel or the base layer packets in spatial domain. The base layer packets contribute more to the overall PSNR quality than the enhancement layer packet. That is, a bigger $k$ protects from the loss of base layer packets, while a smaller $k$ will cause more loss of base layer packets, reducing the PSNR performance more significantly than the loss of enhancement layer packets. With the $E_{b} / N_{0}$ increasing, in the VGA scenario, using $k=1$ offers the best PSNR performance. This is because in the good channel condition, given that very few base layer packets are lost, using $k=1$ means relatively strong protection for the enhancement layer packets, and less loss of enhancement layer packets provide higher performance.

To evaluate the overall quality performance received by the two users, we calculated the average PSNR performance of the VGA and QVGA versions. In this calculation we assumed that the two users' perceptive quality are equally important. According to the definition of PSNR,

$$
\mathrm{PSNR}=10 \log _{10} \frac{I^{2}}{\mathrm{MSE}},
$$

the Mean Squared Error (MSE) is described by

$$
\mathrm{MSE}=\frac{I^{2}}{10^{\mathrm{PSNR} / 10}} .
$$

Thus, the average MSE of the VGA and the QVGA version of the video trailer is described by

$$
\mathrm{MSE}_{\mathrm{ave}}=\frac{\mathrm{MSE}_{1}+\mathrm{MSE}_{2}}{2}=\frac{I^{2} / 10^{\mathrm{PSNR}_{1} / 10}+I^{2} / 10^{\mathrm{PSNR}_{1} / 10}}{2} .
$$




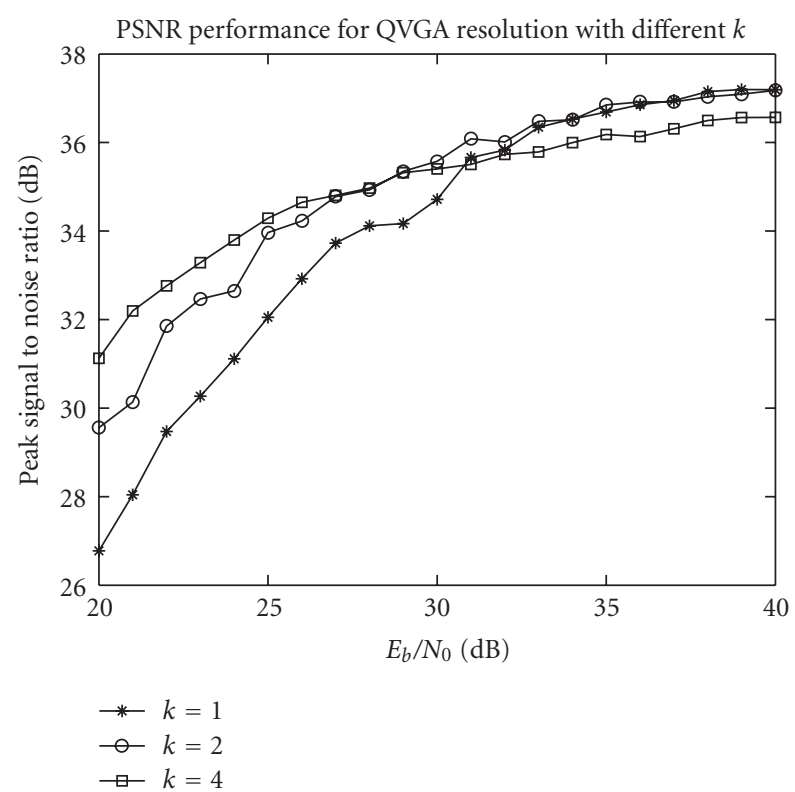

FIGURE 17: The PSNR performance for QVGA resolution with different space ratio $k$. The priority of each data packet is labeled and aware of by the MAC layer.

From (31) and (32) the average PSNR of the VGA and QVGA version of the video is derived to

$$
\begin{aligned}
\operatorname{PSNR}_{\mathrm{ave}} & =10 \log \left(\frac{I^{2}}{\mathrm{MSE}_{\mathrm{ave}}}\right) \\
& =10 \log \left(\frac{2 * 10^{\left(\mathrm{PSNR}_{1}+\mathrm{PSNR}_{2}\right) / 10}}{10^{\mathrm{PSNR}_{1} / 10}+10^{\mathrm{PSNR}_{2} / 10}}\right) .
\end{aligned}
$$

The average PSNR performance of the VGA and QVGA versions is shown in Figure 18.

Note that at low $E_{b} / N_{0}$ using $k=4$ provides the best overall PSNR performance (approximately $6 \mathrm{~dB}$ gain in PSNR at $E_{b} / N_{0}$ of $20 \mathrm{~dB}$ ). When the $E_{b} / N_{0}$ is increasing, using $k=2$ provides a better PSNR performance at the $E_{b} / N_{0}$ in the range of $29 \mathrm{~dB}$ and $35 \mathrm{~dB}$. When the $E_{b} / N_{0}$ is larger than $35 \mathrm{~dB}$, using $k=4$ offers the best PSNR performance. Assuming that the $E_{b} / N_{0}$ can be estimated and known by the transmitter and the receiver, a hierarchical constellation scheme which can provide the best PSNR performance by using a selection of $k$ values is shown in Figure 19.

\section{Conclusion}

This paper proposed an optimized hierarchical modulation strategy directed by cross-layer transport priority information for both single user scenario and two users scenario. In the one user scenario, the hierarchical modulation combined with a convolutional code is designed to achieve the objective bit error rates of two data channels with different priority level by an overall minimum signal to noise ratio. In the two-user scenario, the hierarchical modulation strategy, the video codec scalability in spatial domain is considered and an optimized strategy is proposed for the best perceivable

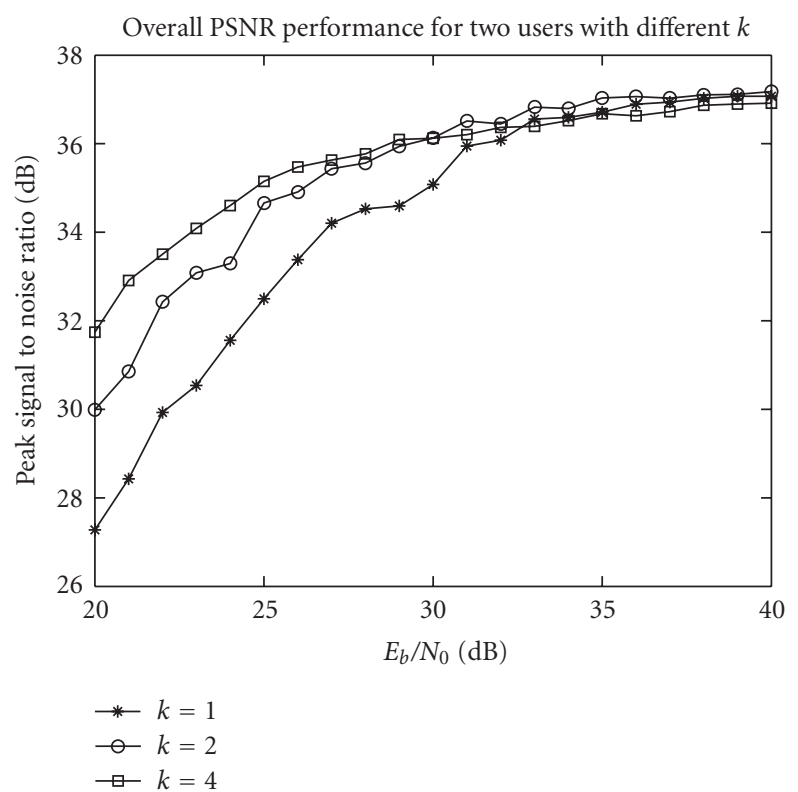

FIGURE 18: The overall PSNR performance for both VGA and QVGA resolutions with different space ratio $k$. The priority of each packet is labeled by and aware of by the MAC layer.

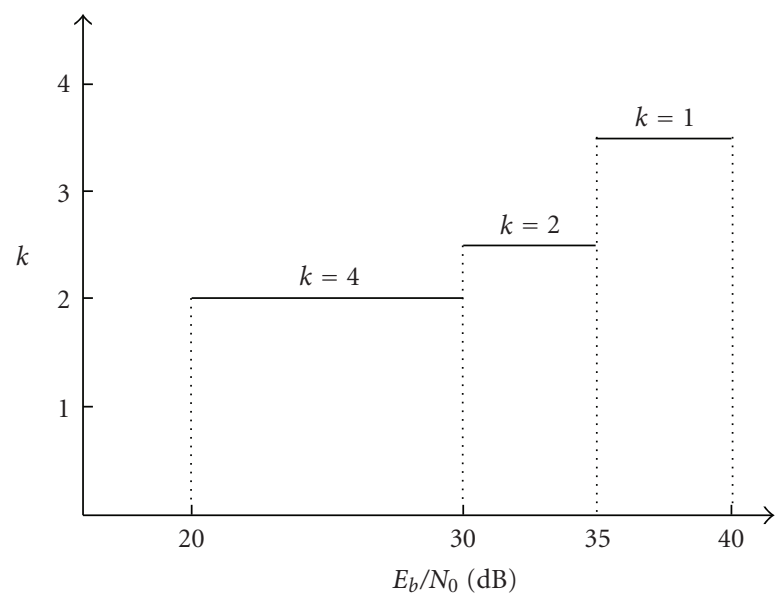

FIgURE 19: A hierarchical constellation scheme for the best overall PSNR performance using a selection of $k$ value.

quality of the scalable video transmission. The optimization strategy can be implemented in the timescale of $1 \mathrm{~ms}$ to $10 \mathrm{~ms}$. The simulation results show $1.5 \mathrm{~dB}$ gain in $E_{b} / N_{0}$ in the single user scenario and $6 \mathrm{~dB}$ gain in PSNR (perceivable quality of the scalable video) in two user scenario. Hierarchical modulation is proved to be a promising candidate for the transmission system for scalable digital media.

\section{Acknowledgment}

The authors would like to thank Krishna Sankar because the inspirations about how to calculate the generic bit error probability over flat Rayleigh fading channel is from his web site http://www.dsplog.com/. 


\section{References}

[1] J.-R. Ohm, "Advances in scalable video coding," Proceedings of the IEEE, vol. 93, no. 1, pp. 42-56, 2005.

[2] A. Lallet, C. Dolbear, J. Hughes, and P. Hobson, "Review of scalable video strategies for distributed video applications," in Distributed Imaging IEE European Workshop, vol. 1999, pp. 2/1-2/7, London, UK, 1999.

[3] H. Schwarz and D. Marpe, "D2.2:documentation of the OpenLoop SNR-Scalable Video Coder," M-PIPE, September 2005, http://cordis.europa.eu/ist/kct/mpipe_synopsis.htm.

[4] M. van der Schaar, S. Krishnamachari, S. Choi, and X. Xu, "Adaptive cross-layer protection strategies for robust scalable video transmission over 802.11 WLANs," IEEE Journal on Selected Areas in Communications, vol. 21, no. 10, pp. 1752 $1763,2003$.

[5] T. M. Cover, "Broadcast channels," IEEE Transactions on Information Theory, vol. 18, pp. 2-14, 1972.

[6] L.-F. Wei, "Coded modulation with unequal error protection," IEEE Transactions on Communications, vol. 41, no. 10, pp. 1439-1449, 1993.

[7] C. E. W. Sundberg, W. C. Wong, and R. Steele, "Logarithmic PCM weighted QAM transmission over gaussian and Rayleigh fading channels," IEE Proceedings, vol. 134, no. 6, pp. 557-570, 1987.

[8] K. Ramchandran, A. Ortega, K. M. Uz, and M. Vetterli, "Multiresolution broadcast for digital HDTV using joint source/channel coding," IEEE Journal on Selected Areas in Communications, vol. 11, no. 1, pp. 6-23, 1993.

[9] M. Morimoto, H. Harada, M. Okada, and S. Komaki, "A study on power assignment of hierarchical modulation schemes for digital broadcasting," IEICE Transactions on Communications, vol. E77-B, no. 12, pp. 1439-1449, 1994.

[10] M. Morimoto, M. Okada, and S. Komaki, "A hierarchical image transmission system in a fading channel," in Proceedings of the 4th IEEE International Conference on Universal Personal Communications (ICUPC '95), pp. 769-772, Tokyo, Japan, November 1995.

[11] M. B. Pursley and J. M. Shea, "Nonuniform phase-shiftkey modulation for multimedia multicast transmission in mobile wireless networks," IEEE Journal on Selected Areas in Communications, vol. 17, no. 5, pp. 774-783, 1999.

[12] M. B. Pursley and J. M. Shea, "Adaptive nonuniform phaseshift-key modulation for multimedia traffic in wireless networks," IEEE Journal on Selected Areas in Communications, vol. 18, no. 8, pp. 1394-1407, 2000.

[13] "DVB-T standard: ETS 300 744, digital broadcasting systems for television, sound and data services; framing structure, channel coding and modulation for digital terrestrial television," The European Telecommunications Standards Institute, vol. 1.5.1, no. EN300, p. 744, 2004.

[14] C. Nokes and J. Mitchell, "Protential benefits of hierarchical modes of the DVB-T specification," in Proceedings of the IEE Colloquium on Digital Television-Where Is It and Where Is It Going? pp. 10/1-10/6, London, UK, 1999.

[15] P. Marsden, "Some thoughts on the use of hierarchical modulation in dvb-t," BBC Research and Development Whiter Paper WHP 028, April 2002.

[16] H. Jiang and P. A. Wilford, "A hierarchical modulation for upgrading digital broadcast systems," IEEE Transactions on Broadcasting, vol. 51, no. 2, pp. 223-229, 2005.
[17] J. Lu, K. B. Letaief, J. C.-I. Chuang, and M. L. Liou, "M-PSK and M-QAM BER computation using signal-space concepts," IEEE Transactions on Communications, vol. 47, no. 2, pp. 181184, 1999.

[18] L.-L. Yang and L. Hanzo, "A recursive algorithm for the error probability evaluation of M-QAM," IEEE Communications Letters, vol. 4, no. 10, pp. 304-306, 2000.

[19] M. P. Fitz and J. P. Seymour, "On the bit error probability of QAM modulation," International Journal of Wireless Information Networks, vol. 1, no. 2, pp. 131-139, 1994.

[20] D. Yoon, K. Cho, and J. Lee, "Bit error probability of Mary quadrature amplitude modulation," in Proceedings of IEEE Vehicular Technology Conference (VTC '00), vol. 5, pp. 24222427, Boston, Mass, USA, September 2000.

[21] P. K. Vitthaladevuni and M.-S. Alouini, "BER computation of 4/M-QAM hierarchical constellations," IEEE Transactions on Communications, vol. 47, no. 3, pp. 228-239, 2001.

[22] J. G. Proakis, Digital Communications, McGraw-Hill, New York, NY, USA, 2001.

[23] S. Rappaport, "Computing approximations for the generalized Q function and its complement," IEEE Transactions on Information Theory, vol. 17, no. 4, pp. 497-498, 1971.

[24] P. Borjesson and C. E. Sundberg, "Simple approximations of the error function $\mathrm{Q}(\mathrm{x})$ for communications applications," IEEE Transactions on Communications, vol. 27, no. 3, pp. 639643, 1979.

[25] S. A. Dyer and J. S. Dyer, "Approximations to error functions," IEEE Instrumentation and Measurement Magazine, vol. 10, no. 6, pp. 45-48, 2007.

[26] P. Van Halen, "Accurate analytical approximations for error function and its integral," Electronics Letters, vol. 25, no. 9, pp. 561-563, 1989.

[27] J. Shapiro, "Embedded image coding using zerotrees of wavelet coefficients," IEEE Transactions on Signal Processing, vol. 41, no. 12, pp. 3445-3462, 1993.

[28] J. Medbo, "Channel models for hiperlan/2 in different indoor scenarios," ETSI BRAN 3ERIO85B, March 1998. 